\title{
Synthesis of Polystyrenesulfonic Stabilized Magnetite Nanoparticles
}

\author{
K. BASAVAIAH* ${ }^{*}$ and A.V. PRASADA RAO \\ Department of Inorganic and Analytical Chemistry, Andhra University, \\ Visakhapatnam -530003, India \\ klbasu@gmail.com
}

Received 28 May 2012 / Accepted 20 June 2012

\begin{abstract}
Superparamagnetic magnetite $\left(\mathrm{Fe}_{3} \mathrm{O}_{4}\right)$ nanoparticles have been synthesized via coprecipitation method in presence of organic sulfonic acid. The product were characterized by x-ray diffraction, UV-visible spectroscopy, fourier transform infrared spectroscopy, transmission electron microscopy and vibrating sampling magnetometer (VSM). X-ray diffraction data indicated that the successfully formation of $\mathrm{Fe}_{3} \mathrm{O}_{4}$ nanoparticles. As obtained $\mathrm{Fe}_{3} \mathrm{O}_{4}$ nanoparticles have spherical shape and size in the range of 5-6 nm without any aggregation of nanoparticles. VSM studies indicated that superparamagnetic properties of $\mathrm{Fe}_{3} \mathrm{O}_{4}$ nanoparticles.
\end{abstract}

Keywords: Magnetite nanoparticles, Co-precipitation, Superparamagnetism, Surfactant

\section{Introduction}

Superparamagnetic magnetite $\left(\mathrm{Fe}_{3} \mathrm{O}_{4}\right)$ nanoparticles have attracted much attention to both fundamental research and technological applications such as targeted drug delivery systems, magnetic separation, magnetic storage media, contrast agents for magnetic resonance image (MRI), bio labelling and separation of biomoelucles ${ }^{1-9}$. All these applications requires the magnetite nanoparticles particles to be chemically stable, have particles size $<20 \mathrm{~nm}$ with a narrow size distribution and to be well dispersed in aqueous medium ${ }^{10}$. However, $\mathrm{Fe}_{3} \mathrm{O}_{4}$ nanoparticles can easily agglomerate due to magnetic dipole-dipole interactions. Numerous approaches have been developed for synthesis of $\mathrm{Fe}_{3} \mathrm{O}_{4}$ nanoparticles using various capping agnets such as silica, gold, polymers and capping agents. Despite a few of papers concerning the preparation of $\mathrm{Fe}_{3} \mathrm{O}_{4}$ nanoparticles and their applications in biomedicines, their practical application is still limited. Usually an external magnetic field was used to suspend paramagnetic particles in biomaterial applications and the resulting materials were expected to do not agglomerate anymore after removal of the field. At present, the significant question is that magnetic particles can easily aggregate in the presence or even the absence of a magnetic field. Furthermore, the major difficulty lies in the interrelated effects in synthesis and assembly. Agglomeration of these particles originates from the strong interaction among the particles, which will diminish their magnetic properties. Therefore, it is of fundamental importance to develop a method to synthesize control size and shape $\mathrm{Fe}_{3} \mathrm{O}_{4}$ nanoparticles without any agglomeration. 
In this paper, we present the synthesis of polystyrene sulfonic acid (PSS) stabilized $\mathrm{Fe}_{3} \mathrm{O}_{4}$ nanoparticles via co-precipitation method. The effect of PSS content on magnetic properties of $\mathrm{Fe}_{3} \mathrm{O}_{4}$ nanoparticles was investigated.

\section{Experimental}

Polystyrene sulfonic acid received from Aldrich and was used as received. Other reagents such as $\mathrm{FeCl}_{3} \cdot 6 \mathrm{H}_{2} \mathrm{O}, \mathrm{FeSO}_{4} \cdot 7 \mathrm{H}_{2} \mathrm{O}$, ammonia $\left(\mathrm{NH}_{3} \mathrm{H}_{2} \mathrm{O}\right)$, sodium hydroxide and methanol were purchased from Merck, India and used as received without any purification. Double distilled water was used throughout the synthetic processes. All other reagents were analytical grade and used without further purification.

\section{Synthesis of PSS stabilized $\mathrm{Fe}_{3} \mathrm{O}_{4}$ nanoparticles}

The PSS stabilized $\mathrm{Fe}_{3} \mathrm{O}_{4}$ nanoparticles were synthesized by a self-assembly method in presence of PSS via modified co-precipitation method. In a typical synthesis process, a $5 \mathrm{~mL}$ of PSS dissolved in $50 \mathrm{~mL}$ of distilled water under vigorous stirring at room temperature. The reaction mixture was constantly stirring for 2 hours to obtain a milky dispersion of PSS micelles. Then, $10 \mathrm{~mL}$ of $1 \mathrm{~mol} \mathrm{~L}^{-1} \mathrm{FeCl}_{3} \cdot 6 \mathrm{H}_{2} \mathrm{O}$ and $10 \mathrm{~mL}$ of 0.5 mol L-1 $\mathrm{FeSO}_{4} \cdot 7 \mathrm{H}_{2} \mathrm{O}$ aqueous solutions were rapidly injected into the above reaction mixture and slowly heated up to $65{ }^{\circ} \mathrm{C}$ under stirring, followed by the addition of $5 \mathrm{~mL}$ of $25 \% \mathrm{NH}_{4} \mathrm{OH}$. The colour of reaction mixture turns black indicating the formation of magnetite. The reaction was allowed to proceed for $5 \mathrm{~h}$ under vigorous stirring until a very viscous PSS stabilized magnetite nanoparticles were obtained. The resulting mixture was centrifugated and washed with distilled water and finally with methanol. The product was then dried under vacuum at room temperature for $12 \mathrm{~h}$. The same procedure has been adopted for preparation of all PSS stabilized $\mathrm{Fe}_{3} \mathrm{O}_{4}$ nanoparticles, while varying the PSS content in the reaction mixture.

\section{Characterization}

The UV-Visible absorption spectra of the samples were recorded on a Perkin-Elmer double beam LS-50 spectrophotometer. The morphology, chemical composition and phase identification of $\mathrm{Fe}_{3} \mathrm{O}_{4} / \mathrm{PANI}$ nanocomposites were characterized by scanning electron microscopy (SEM), energy dispersive X-ray spectroscopy (EDX), transmission electron microscopy (TEM) and x-ray diffraction (XRD). Powder $\mathrm{x}$-ray diffraction (XRD) pattern were recorded on a Siemens D5005 x-ray diffractometer at 1 per min. Magnetic properties were investigated by vibrating sample magnetometer (VSM, Lake Shore, USA).

\section{Results and Discussion}

The synthesis of $\mathrm{Fe}_{3} \mathrm{O}_{4}$ nanoparticles is based on modified co-precipitation chemical in the presence of excess of PSS as soft-template. The template, PSS is believed to serve as the nanoreactor. Because of its hydrophilic group $\left(-\mathrm{SO}_{3} \mathrm{H}\right)$ and hydrophobic group $\left(-\mathrm{C}_{12} \mathrm{H}_{25}\right)$, PSS easily forms micelles in an aqueous solution. Micelle-assisted synthesis had previously been used widely to prepare nano particles in which the particle sizes were controlled by the micelle "reactor" sizes" ${ }^{11}$. The presence of excess PSS can prevent the formation of larger particles via steric hindrance and thereby producing nanometer sized nanoparticles. $\mathrm{Fe}_{3} \mathrm{O}_{4}$ nanoparticles were also formed inside PSS micelles "reactor" during the sedimentation process. PSS having $-\mathrm{SO}_{3}^{-}$group can stabilize $\mathrm{Fe}_{3} \mathrm{O}_{4}$ nanoparticles, while long alkyl hydrophobic chains act as a barrier to protect the particles from agglomeration ${ }^{12}$. 
Molecular structure and phase identification was characterized by x-ray diffraction pattern (XRD) and UV-Visible spectroscopy. Figure 1 show the XRD patterns from PSS stabilized $\mathrm{Fe}_{3} \mathrm{O}_{4}$ nanoparticles. The diffraction peaks at $2 \theta=30.47^{\circ}, 35.63^{\circ}, 43.35^{\circ}, 57.22^{\circ}$, $63.06^{\mathrm{o}}$ can be indexed as (220), (311), (400), (511) and (440) planes of magnetite, respectively.

Figure 2 depicts the UV-Visible spectra of PSS stabilized $\mathrm{Fe}_{3} \mathrm{O}_{4}$ nanoparticles which is in complete agreement with literature reports ${ }^{13}$. The UV-Visible absorption spectrum showed a characteristic absorbance band at $304 \mathrm{~nm}$. Besides this peak, there is a sharp peak at $290 \mathrm{~nm}$ due to the $\pi \rightarrow \pi^{*}$ transition in the benzenoid rings of PSS, which confirms the capping of PSS to the surface of $\mathrm{Fe}_{3} \mathrm{O}_{4}$ nanoparticles.

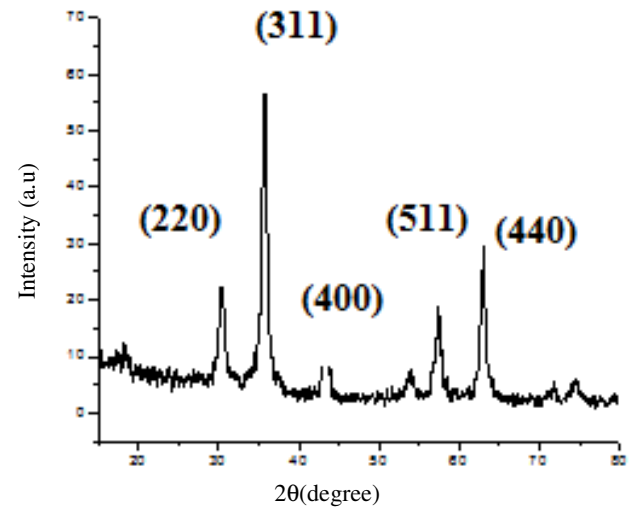

Figure 1. The XRD patterns from PSS stabilized $\mathrm{Fe}_{3} \mathrm{O}_{4}$ nanoparticles

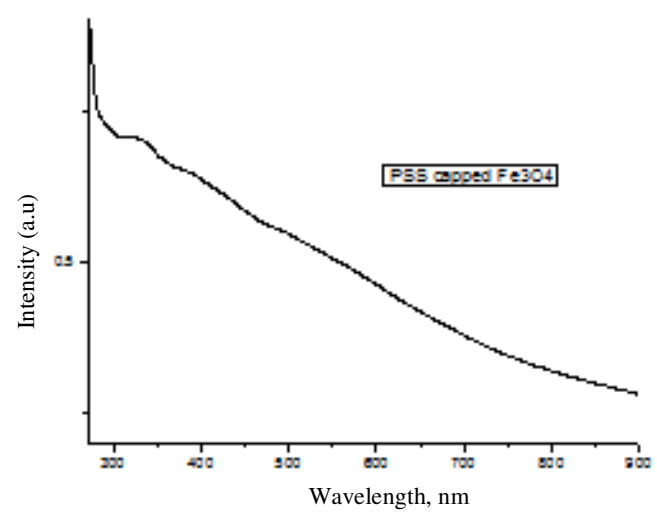

Figure 2. The UV-Visible spectra of PSS stabilized $\mathrm{Fe}_{3} \mathrm{O}_{4}$ nanoparticles

According to the reflections peaks shown in Figure $1 \& 2$, the main phases can be indexed as magnetite, which are in good agreement with the standard magnetite card (card No. PDF\#01-1111). The XRD patterns of PSS stabilized $\mathrm{Fe}_{3} \mathrm{O}_{4}$ nanoparticle is indicated that the crystalline in nature and completely agree with previous literature.

The morphology of PSS stabilized $\mathrm{Fe}_{3} \mathrm{O}_{4}$ nanoparticles have been investigated by scanning electron microscopy. Figure 3 shows the scanning electron microscope (SEM) image of $\mathrm{Fe}_{3} \mathrm{O}_{4}$ nanoparticles prepared via co-precipitation method in presence of PSS. It can be clearly seen from $\mathrm{SEM}$ image that $\mathrm{Fe}_{3} \mathrm{O}_{4}$ nanoparticles were of sphere like morphology.

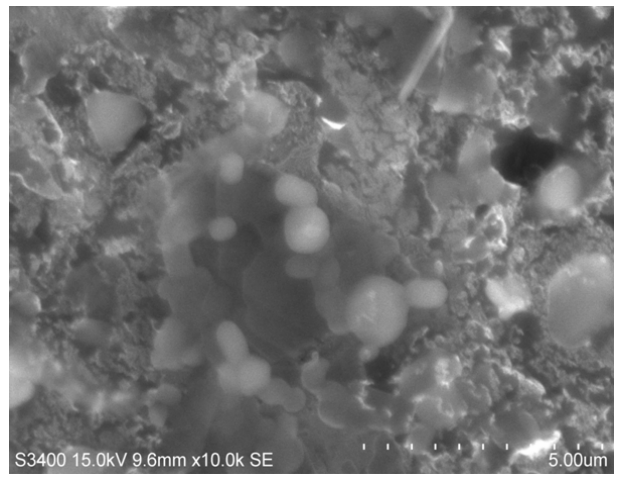

Figure 3. SEM image of PSS stabilized $\mathrm{Fe}_{3} \mathrm{O}_{4}$ nanoparticles 
Transmission electron microscopy (TEM) image of as prepared PSS stabilized $\mathrm{Fe}_{3} \mathrm{O}_{4}$ nanoparticles displayed in Figure 4, shows $\mathrm{Fe}_{3} \mathrm{O}_{4}$ nanoparticles are nearly spherical with an average diameter ranges from 5-6 $\mathrm{nm}$. It can be seen that the vast majority of particles exhibit a diameter close to 5-6 nm without particles aggregation, because as synthesized $\mathrm{Fe}_{3} \mathrm{O}_{4}$ nanoparticles were coated with PSS.
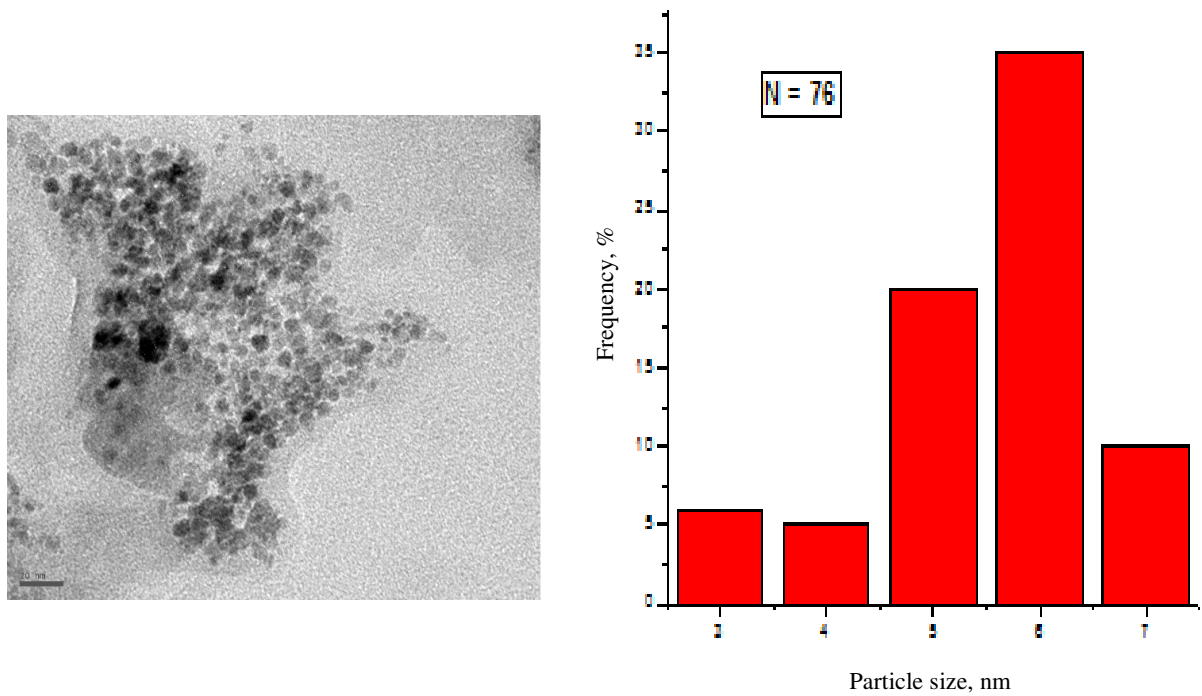

Figure 4. Representative TEM image and particle size of $\mathrm{Fe}_{3} \mathrm{O}_{4}$ nanoparticles prepared by modified co-precipitation method.

To investigate the influence of the PSS on magnetic properties of $\mathrm{Fe}_{3} \mathrm{O}_{4}$ nanoparticles and hysteresis loops of samples prepared at different content of PSS were analyzed by vibrating sampling magnetometer (VSM), as shown in Figure 5. The magnetization curve shows no remanence or coercivity at room temperature, suggesting superparamagnetic behaviour with saturation magnetization values: $11,9,2.3,2.5$ and $2 \mathrm{emu} / \mathrm{g}$ for the samples prepared at different content of PSS. These Ms values were much more lower than bulk $\mathrm{Fe}_{3} \mathrm{O}_{4}$ value ${ }^{14}$.

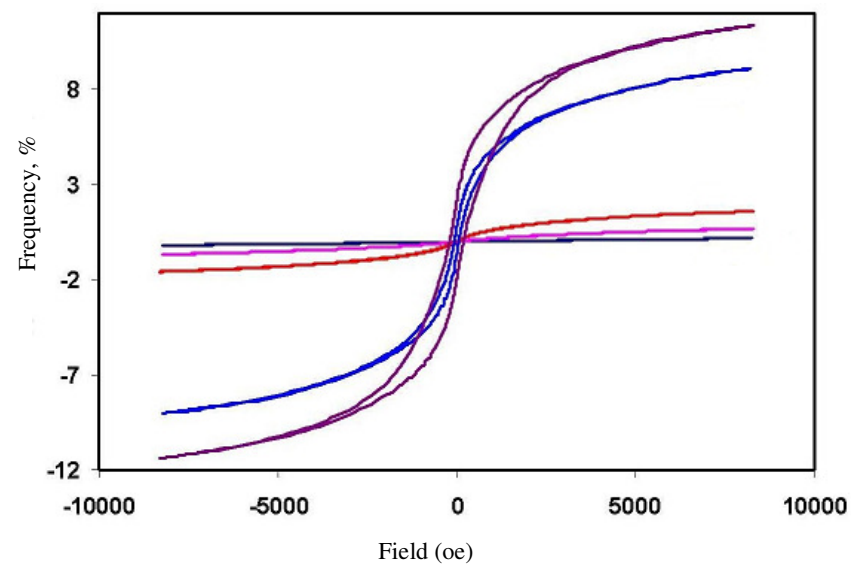

Figure 5. Room temperature magnetization curves of $\mathrm{Fe}_{3} \mathrm{O}_{4}$ nanoparticles prepared at different content of PSS 
It can be observed that the magnetic saturation $\left(\mathrm{M}_{\mathrm{s}}\right)$ values of $\mathrm{Fe}_{3} \mathrm{O}_{4}$ nanoparticles were decreased by increasing content of non magnetic PSS coating on $\mathrm{Fe}_{3} \mathrm{O}_{4}$ nanoparticles. The changes in the magnetic properties of the samples can be attributed to the modification of the crystallinity and crystallite sizes dependent on the content of PSS. The energy of a magnetic particle in the external field is proportional to its particle sizes via the number of molecules in a single magnetic domain. Therefore, the decrease of the $\mathrm{M}_{\mathrm{s}}$ values with the decrease of particle sizes can be attributed to surface effects that are the result of finite-size scaling of nanocrystallites ${ }^{15-16}$.

\section{Conclusion}

In summary, we have demonstrated a simple, reproducible and facile method of preparation of $\mathrm{Fe}_{3} \mathrm{O}_{4}$ nanoparticles via co-precipitation method in presence of PSS. The spectroscopic and XRD results indicated that the successfully formation of agglomerate free $\mathrm{Fe}_{3} \mathrm{O}_{4}$ nanoparticles. $\mathrm{Fe}_{3} \mathrm{O}_{4}$ nanoparticles have superparamagnetic nature with much lower saturation magnetization than bulk magnetite.

\section{Acknowledgment}

The author would like to thank the Ministry of Earth Science (MOES, India), India and also University Grant Commission, India (UGC, India) for financial support of this work. We are also thankful to Prof. T. P. Radhakrishnan and M.V. Prasad, Centre for nanotechnology, University of Hyderabad for providing TEM facility.

\section{References}

1. Jun YW, Seo J W and Cheon J, Acc Chem Res., 2008, 41(2), 179.

2. Xie J, Peng S, Brower N, Pourmand N, Wang S X and Sun S, Pure Appl Chem., 2006, 78(5), 1003-1014.

3. Xu C and Sun S, Polym Int., 2007, 56, 821.

4. Lewinski N, Colvin V and Drezek R, Small., 2008, 4(1), 26-49.

5. Xie J, Xu C, Xu Z, Hou Y, Young K L, Wang S X, Pourmand N and Sun S, Chem Mater., 2006, 18(23), 5401-5403.

6. Pankhurst Q A, Connolly J, Jones S K and Dobson J, J Phys D Appl Phys., 2003, 36, R167-R181.

7. McNeil S, J Leukocyte Biol., 2005, 78, 585-594.

8. Xu C, Xie J, Kohler N, Walsh E G, Chin Y E and Sun S, Chem Asian J., 2008, 3(3), 548-552.

9. Sun S and Zeng H, J Am Chem Soc., 2002, 124, 8204-8205.

10. Gupta A K and Gupta M, Biomaterials, 2005, 26(18), 3995-4021.

11. Kinlen P J, Liu J, Ding Y, Graham C R and Remsen E E, Macromolecules, 1998, 31, 1735-1744.

12. Hagerstrand H, Bobacka J, Hagerstrand M B, Iglic V K, Fosnaric M and Iglic A, Cellular Molecular Bio Lett., 2001, 6, 161-165.

13. Kohler N, Sun C, Fichtenholtz A, Gunn J, Fang C and Zhang M, Small., 2006, 2(6), 785-792.

14. Coey J M D, Morrish A H and Sawatzky G A, J Phys., 1971, 32 C1, 271-273.

15. Mohallem N D S and Seara L M, Appl Surf Sci., 2003, 214, 143-150.

16. Gee S H, Hong Y K, Erickson D W and Park M H, J Appl Phys., 2003, 93, 7560-7562. 